ARTICLE

DOI: $10.1038 / \mathrm{s} 41467-018-03794-9$

\title{
Experimental determination of the energy difference between competing isomers of deposited, size-selected gold nanoclusters
}

\author{
D.M. Foster ${ }^{1}$, R. Ferrando ${ }^{2} \&$ R.E. Palmer (iD ${ }^{3}$
}

The equilibrium structures and dynamics of a nanoscale system are regulated by a complex potential energy surface (PES). This is a key target of theoretical calculations but experimentally elusive. We report the measurement of a key PES parameter for a model nanosystem: size-selected Au nanoclusters, soft-landed on amorphous silicon nitride supports. We obtain the energy difference between the most abundant structural isomers of magic number $\mathrm{Au}_{561}$ clusters, the decahedron and face-centred-cubic (fcc) structures, from the equilibrium proportions of the isomers. These are measured by atomic-resolution scanning transmission electron microscopy, with an ultra-stable heating stage, as a function of temperature $\left(125-500^{\circ} \mathrm{C}\right)$. At lower temperatures $\left(20-125^{\circ} \mathrm{C}\right)$ the behaviour is kinetic, exhibiting down conversion of metastable decahedra into fcc structures; the higher state is repopulated at higher temperatures in equilibrium. We find the decahedron is $0.040 \pm 0.020 \mathrm{eV}$ higher in energy than the fcc isomer, providing a benchmark for the theoretical treatment of nanoparticles.

\footnotetext{
${ }^{1}$ Nanoscale Physics Research Laboratory, School of Physics and Astronomy, University of Birmingham, Birmingham B15 2TT, UK. ${ }^{2}$ Chemistry and Industrial Chemistry Department, University of Genoa, Via Dodecaneso 31, 16146 Genoa, Italy. ${ }^{3}$ College of Engineering, Swansea University, Bay Campus, Fabian Way, Swansea SA1 8EN, UK. Correspondence and requests for materials should be addressed to R.E.P. (email: R.E.Palmer@swansea.ac.uk)
} 
T he structure and dynamics of a nanosystem are controlled by the multi-dimensional potential energy surface (PES), which describes its free energy as a function of configuration. There have been considerable theoretical efforts to determine the ground-state structures and energy differences between competing isomers of nanosytems in general ${ }^{1-3}$ and of nanoclusters in particular ${ }^{4-8}$. Gold clusters have received much theoretical attention due to the role of structure in the catalytic performance ${ }^{9}$. What is needed now is an experimental handle on key parameters of the PES. Understanding the energy difference between structural isomers is important not only for the design of well-defined materials but also for understanding how these materials will work in situ. For example, if a particular structural isomer is unstable, exposure to high temperatures is likely to drive it towards the ground state (i.e. annealing), altering (for better or worse) the characteristics of the system. Such behaviour is likely to be relevant to the applications of nanoparticles, which include catalysis ${ }^{10,11}$, drug delivery ${ }^{12,13}$ and chemical sensing ${ }^{14}$.

Experimentally the atomic structure of nanoclusters can be determined, to various degrees, by a number of techniques including trapped ion electron diffraction ${ }^{15}$, x-ray scattering ${ }^{16}$, transmission electron microscopy (TEM) tilt series ${ }^{17}$ and highangle annular dark-field (HAADF) aberration-corrected scanning transmission electron microscopy (ac-STEM) ${ }^{18}$. However, the cluster formation conditions can easily lead to the trapping of higher lying isomers and the populations of cluster isomers observed do not represent thermal equilibrium ${ }^{19}$. Previous STEM studies have provided some qualitative insight into the PES of clusters through e-beam transformation experiments. By continual imaging during intense irradiation, $\mathrm{Au}_{561}{ }^{20}$ and $\mathrm{Au}_{923}{ }^{19}$ clusters (on carbon) have been shown to transform one-way to lower energy structures ${ }^{19}$, while smaller clusters fluctuate continually ${ }^{21-23}$. Such experiments enable candidate low energy structures to be identified. However, these experiments do not provide the quantitative energy difference between isomers. Ex situ annealing experiments by Koga et al. ${ }^{24}$ found that annealing of small and medium sized $(<14 \mathrm{~nm})$ Au clusters below the melting point $(<1273 \mathrm{~K})$ resulted in structural transformations, but no quantitative measure of energy differences or barrier heights could be made.

Here we employ a precision heating stage in ac-STEM to determine in situ the proportion of structural isomers for sizeselected $\mathrm{Au}_{561}$ clusters, deposited on amorphous silicon nitride, over a range of temperatures. This enables the energy difference between competing fcc and $\mathrm{Dh}$ isomers in the equilibrium region to be extracted for $\mathrm{Au}_{561}$ on the surface. We identify two regimes: a low-temperature regime in which metastable (kinetically trapped) Dh clusters transform to fcc, and a high-temperature regime in which the $\mathrm{Dh}$ isomer is repopulated (Boltzmann statistics); here the system is in thermal equilibrium. From the equilibrium, high-temperature region data, we find that the $\mathrm{Dh}$ and fcc isomers are very close in energy, where the Dh are only $0.040 \pm$ $0.020 \mathrm{eV}$ higher than those of fcc.
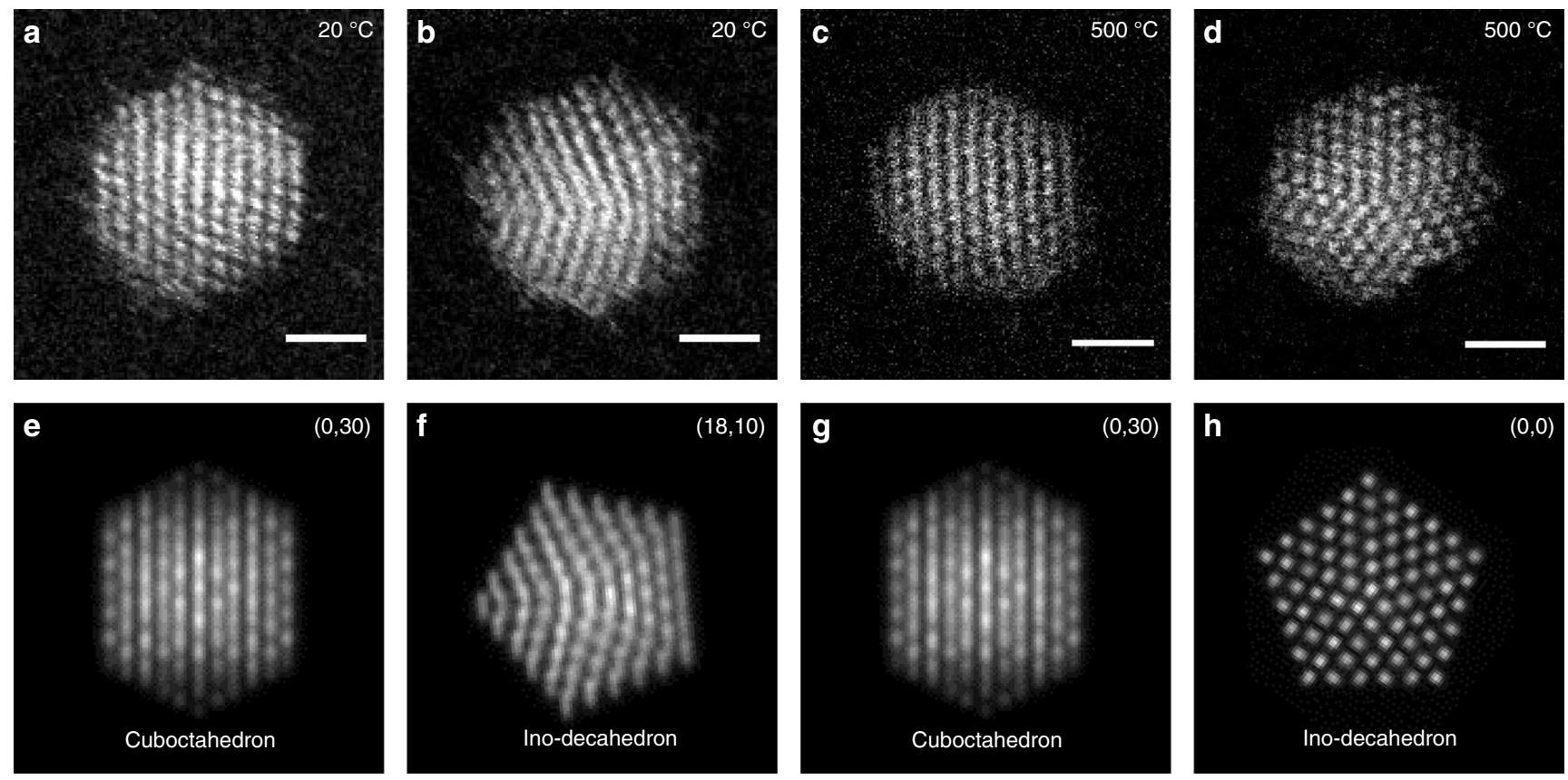

i
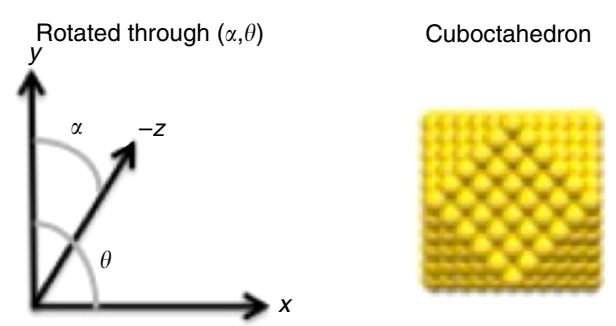

Ino-decahedron

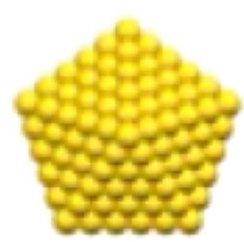

Fig. $1 \mathrm{HAADF}$ STEM images of Au $\mathrm{H}_{51}$ clusters at $20^{\circ} \mathrm{C}$ and $500^{\circ} \mathrm{C}$. a-d HAADF STEM images of Au $u_{561}$ clusters and $\mathbf{e}-\mathbf{h}$ matching multi-slice electron scattering simulations of the cuboctahedron and Ino-decahedron at different orientations. a, b Experimental images recorded at $20^{\circ} \mathrm{C} ; \mathbf{c}$, $\mathbf{d}$ Images recorded at $500^{\circ} \mathrm{C}$. i Rotation angle of the cuboctahedron and Ino-decahedron geometries 

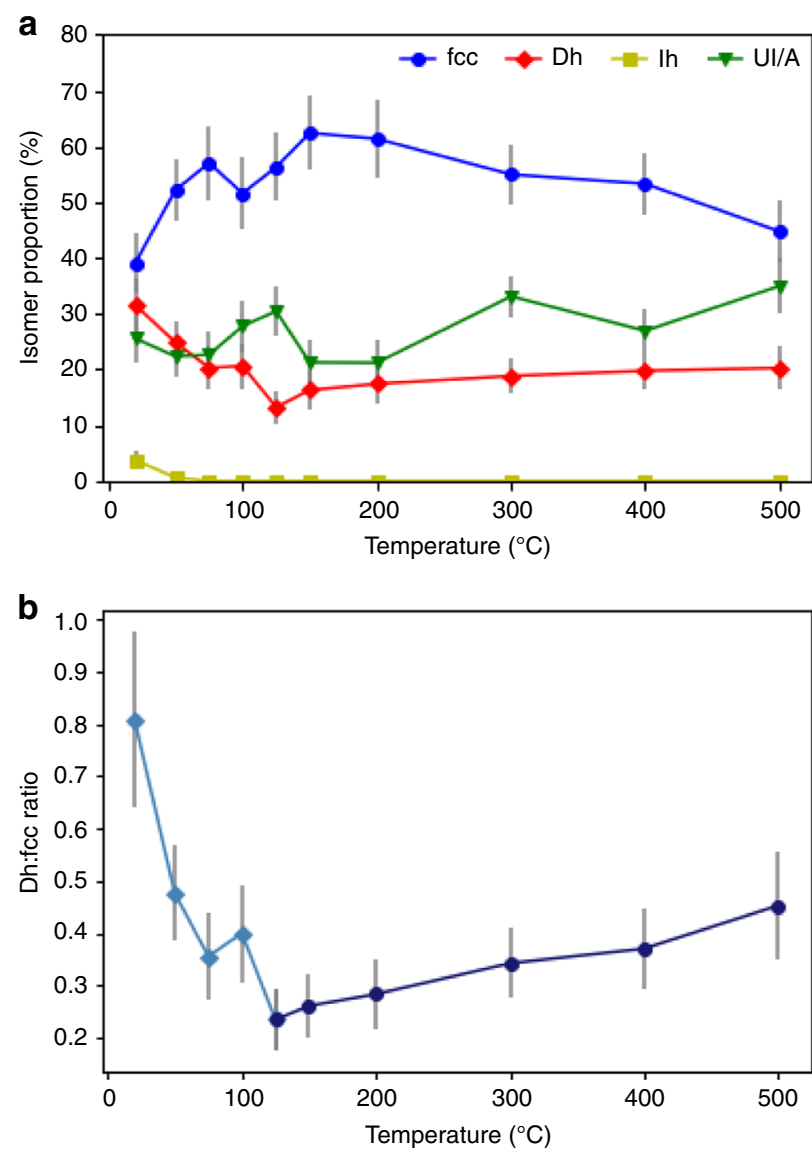

Fig. 2 The proportion of structural isomers versus temperature. a The proportion of structural isomers for $\mathrm{Au}_{561}$ clusters on amorphous silicon nitride at ten temperatures: $20^{\circ} \mathrm{C}, 50^{\circ} \mathrm{C}, 75^{\circ} \mathrm{C}, 100^{\circ} \mathrm{C}, 125^{\circ} \mathrm{C}, 150^{\circ} \mathrm{C}$, $200^{\circ} \mathrm{C}, 300^{\circ} \mathrm{C}, 400^{\circ} \mathrm{C}$ and $500^{\circ} \mathrm{C}$. The clusters are classified as facecentred-cubic (circles), decahedral (diamonds), icosahedral (squares) or unidentified/amorphous (triangles). The numbers of experimental images recorded at each temperature are 133, 161, 128, 126, 151, 141, 132, 191, 167 and 143 respectively. Poisson error bars, derived from these statistics, are shown for the isomer proportions. $\mathbf{b}$ The ratio of $\mathrm{Dh}$ to fcc clusters versus temperature. The low-temperature regime $\left(20-125^{\circ} \mathrm{C}\right)$ is in diamond markers and the high-temperature regime $\left(125-500^{\circ} \mathrm{C}\right)$ in circle markers. Lines between points plotted are simply a guide to the eye. Error bars, derived according to the error propagation law, are shown for the Dh:fcc ratio

\section{Results}

Electron microscope images. Figure 1 shows examples of HAADF STEM images of $\mathrm{Au}_{561}$ clusters on amorphous silicon nitride and corresponding multi-slice simulations from a simulation atlas ${ }^{19}$. Figure $1 \mathrm{a}$ and $\mathrm{b}$ was recorded at $20^{\circ} \mathrm{C}$. Figure $1 \mathrm{a}$ shows an fcc cluster and Fig. $1 \mathrm{~b}$ shows a decahedral cluster. Figure $1 \mathrm{c}$ and $\mathrm{d}$ was recorded at $500{ }^{\circ} \mathrm{C}$. Figure $1 \mathrm{c}$ shows an $\mathrm{fcc}$ cluster and Fig. 1d an on-axis decahedron. Both decahedra in Fig. $1 \mathrm{~b}$ and $\mathrm{d}$ show some Marks reentrant features. In comparison of experimental and simulated images, we concentrate on the core atomic structure because this is where the signal-to-noise levels are the highest, so that we can compare them with simulations of perfect cuboctahedra and Ino-decahedra. HAADF STEM images matched to the cuboctahedron simulations are denoted facecentred-cubic (fcc), which allows for variation in the exact surface truncation; similarly images matched to the Ino-decahedron are denoted decahedra (Dh). Clusters that display 'ring-dot' features in the images, a characteristic of an icosahedron, are denoted simply as icosahedra (Ih).

Proportions of different isomers. Figure $2 \mathrm{a}$ is a plot of the proportions of structural isomers, extracted from the fits to the experimental data, for $\mathrm{Au}_{561}$ clusters on amorphous silicon nitride at temperatures ranging from $20^{\circ} \mathrm{C}$ to $500^{\circ} \mathrm{C}$. The same sample was used for all measurements so that formation conditions would not affect the results ${ }^{25}$. Cluster structures are identified as either fcc, Dh, Ih or unidentified/amorphous (UI/A). The error bars on the proportions of structural isomers are statistical counting errors and the error on the temperature is $5 \%$, due to the heating chip calibration. At all temperatures investigated the most abundant isomer is fcc, followed by $\mathrm{Dh}$, while Ih has a very low abundance $(0-3 \%)$. We find that the clusters still provide a good match with the simulated structures at high temperature and there is no evidence of melting in the temperature range explored here, as can be seen from Fig. 1. The percentage of unidentified or unknown (UI/A) structures-clusters that are amorphous or cannot be identified using simulation atlases for the Ino-decahedron, cuboctahedron or icosahedron-is fairly constant across the temperature range. One explanation for such images is that only single-shot data was taken (to minimise the electron dose), and clusters often rotate during scanning.

Figure $2 \mathrm{~b}$ shows a plot of the ratio of the two most abundant ordered isomers, $\mathrm{Dh}$ and $\mathrm{fcc}$, versus temperature. Two distinct temperature regimes are clearly visible. Between $20^{\circ} \mathrm{C}$ and $125^{\circ} \mathrm{C}$ the Dh:fcc ratio decreases from 0.81 to 0.24 , whereas between 125 ${ }^{\circ} \mathrm{C}$ and $500{ }^{\circ} \mathrm{C}$ the Dh:fcc ratio increases from 0.24 to 0.45 . The underlying and associated errors are derived from Fig. 2a. Between $20^{\circ} \mathrm{C}$ and $150{ }^{\circ} \mathrm{C}$ the increase in temperature results in an increase in the abundance of the fcc isomer, but at temperatures $\geq 150{ }^{\circ} \mathrm{C}$ the proportion of fcc gradually decreases again. Complementary to this, between $20^{\circ} \mathrm{C}$ and $125^{\circ} \mathrm{C}$, the proportion of $\mathrm{Dh}$ decreases, whereas at temperature $\geq 125^{\circ} \mathrm{C}$ there is a slight increase in Dh as temperature rises.

The increase in the proportion of fcc clusters from $20^{\circ} \mathrm{C}$ to $125^{\circ} \mathrm{C}$, and the corresponding decrease in the proportion of $\mathrm{Dh}$, can be explained in terms of the release of trapped metastable Dh structures to a lower free energy fcc structure. We previously reported that $\mathrm{Au}_{561}$ clusters undergo a one-way transition from $\mathrm{Dh}$ to fcc when continuously exposed to the STEM electron beam at very high magnification ${ }^{20}$, which corresponds to moderate heating of the sample. However, the behaviour we observe takes on a new character above $125^{\circ} \mathrm{C}$ with the ratio of $\mathrm{Dh}$ to fcc increasing again.

This repopulation behaviour can be understood if the fcc structure is a lower free-energy structure than the Dh. Then, beyond the release of kinetically trapped $\mathrm{Dh}$ clusters by annealing at temperatures from $20^{\circ} \mathrm{C}$ to $125^{\circ} \mathrm{C}$, we may expect that an equilibrium distribution of isomers will be established at higher temperatures. A proportion of the clusters (based on Boltzmann statistics) will be excited from the fcc to the higher energy Dh structure $^{26}$. In fact, if we assume equilibrium between isomers of energy $E_{\mathrm{Dh}}$ and $E_{\mathrm{fcc}}$, we obtain the ratio between the probabilities $p_{\mathrm{Dh}}$ and $p_{\mathrm{fcc}}$ of the corresponding structures given by (see the Supplementary Note 1 for a derivation of this formula)

$$
\ln \left(p_{\mathrm{Dh}} / p_{\mathrm{fcc}}\right)=\beta\left(E_{\mathrm{fcc}}-E_{\mathrm{Dh}}\right)+c
$$

where $\beta=\left(k_{\mathrm{B}} T\right)^{-1}$.

In this system the Ih must have much higher energy, as we do not see repopulation of this isomer even at $500^{\circ} \mathrm{C}$; this is in agreement with experimental observations of Ih $\mathrm{Au}_{923}$ clusters under the electron beam, which transformed to Dh or fcc structures after very short exposure times ${ }^{19}$. If the increase in the 


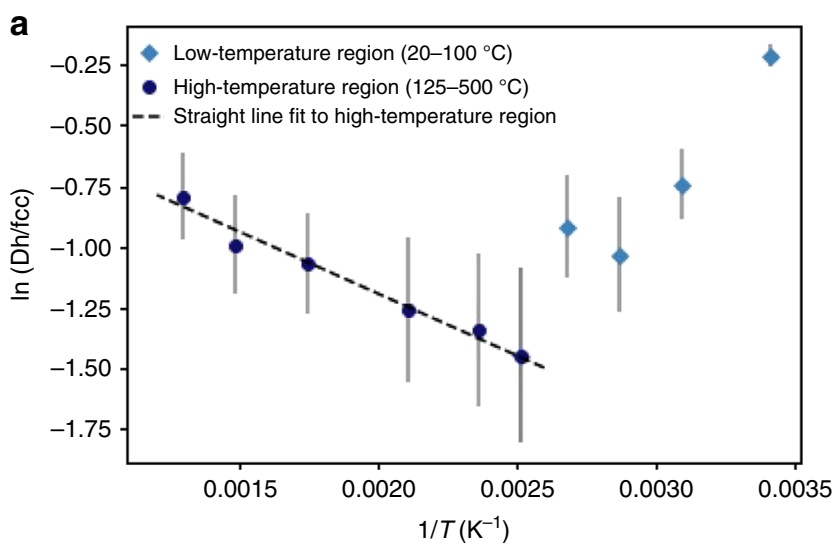

b

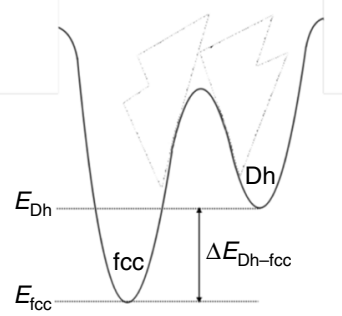

Fig. 3 The derived energy difference between fcc and Dh minima. a Ratio of the $\mathrm{Dh}$ to fcc abundances (natural log plot) for $\mathrm{Au}_{561}$ plotted against the reciprocal of the temperature (in Kelvin). The dashed line shows a weighted least squares fit to the high-temperature region. The equation of this line is: $y=m x+c$ where $m=-510240 K$ and $c=-0.20 \pm 0.40$ in units of $k_{B}$. Error bars, derived from data in Fig. 2 and using error propagation laws, are shown for the natural log of the Dh:fcc ratio. $\mathbf{b}$ A schematic of the derived energy difference between $\mathrm{fcc}$ and Dh minima

proportion of $\mathrm{Dh}$ clusters in the high-temperature region is a result of thermal repopulation of this excited state, the energy difference between the $\mathrm{Dh}$ and fcc structural isomers can be derived, as we show below. A second hypothetical explanation for the change in ratio is that, as the temperature of the clusters increase, atoms are lost through sublimation resulting in a smaller cluster size at higher temperatures where the decahedron might in principle be more stable. However, based on analysis of the diameters of the clusters at $500{ }^{\circ} \mathrm{C}$ (Fig. 1), we are confident that no major loss of atoms has occurred.

Figure 3 shows a plot of the natural log of the ratio of the Dh and fcc abundances as a function of the reciprocal of the absolute temperature. From Eq. (1), the slope of the line in the higher temperature equilibrium regime gives the energy difference between the local minima of the two competing isomers, whereas the intercept gives the entropy difference (see Supplementary Note 1 for detailed explanation). This does not apply to the low temperature, kinetic regime. The dashed line shows a weighted linear least squares fit to the high-temperature region (398-773 $\mathrm{K})$ of the plot. The gradient of this line is $-510 \pm 240 \mathrm{~K}$, which corresponds to a value of $0.040 \pm 0.020 \mathrm{eV}\left(E=k_{\mathrm{B}} T\right)$ for the energy difference between $\mathrm{Dh}$ and the lower lying fcc isomers $\left(\Delta E_{\mathrm{Dh}-\mathrm{fcc}}\right)$. The intercept $c=-0.2 \pm 0.4$ is the entropy difference in units of $k_{\mathrm{B}}$ (Supplementary Note 1), which indicates a negligible entropy difference between these structures.

\section{Discussion}

There are two key assumptions that underpin these new results. First, the partition function for each isomer is given by the harmonic superposition approximation, in which the vibrational frequencies are assumed to be harmonic and independent of temperature. In many cases this approximation has been shown to be valid for temperatures below the melting point ${ }^{8}$. If the vibrational frequencies are anharmonic, there would be a temperature dependence ${ }^{8}$, possibly resulting in non-linearity in the plot of $\ln (\mathrm{Dh} / \mathrm{fcc})$ versus $1 / T$. Secondly, we have assumed that for each basin (Dh-basin, fcc-basin) in the PES of the cluster, there is only a contribution from one structural isomer. In the experiment we have a small range of cluster sizes (determined by the mass filter resolution), and within the classification of $\mathrm{Dh}$ or fcc there may be different truncations and arrangements of atoms on the surface that are not easily distinguished by our simulation atlas method. If whole 'families' of $\mathrm{Dh}$ and fcc isomers are being observed experimentally, the energy difference determined may represent a sort of weighted average of the energy differences between the $\mathrm{Dh}$ and fcc clusters in the families. However, in this case there would not be any compelling reason for obtaining the linear increase shown in Fig. 3 for high temperatures.

The derived energy difference of $0.04 \mathrm{eV}$ between the $\mathrm{Dh}$ and fcc isomers is very small (corresponding to only $\approx 510 \mathrm{~K}$ ), and means that at the cluster size investigated, $561 \pm 14$, these isomers compete very closely. Of course it is the closeness in energy that makes the energy difference nicely measureable in our experiment, which probes a temperature range of $375^{\circ} \mathrm{C}\left(125-500^{\circ} \mathrm{C}\right)$. Regarding the derived energy difference, it would be appealing at this point to produce a theoretical calculation that predicts an energy offset comparable with the experimental value. But the truth is that no calculations currently offer accuracy at the level of tens of $\mathrm{meV}$ for hundreds of atoms! However, the result is in broad qualitative agreement with several ${ }^{27-29}$ theoretical calculations that predict $\mathrm{Dh}$ and fcc isomers competing in energy at this size range. The original molecular dynamic simulations for $\mathrm{Au}$ clusters by Baletto et al. ${ }^{27}$ used the second-moment tightbinding potential for a detailed study and EAM potentials to determine general trends in the energetics of icosahedra, decahedra and truncated octahedral clusters. They found a crossover size from Dh to fcc at 500 atoms, above which the Dh and fcc isomers remain close in energy, whilst the Ih is not favoured above 100 atoms. More recently, Wang et al. ${ }^{28}$ also found that fcc is the lowest energy structure for clusters with more than 500 atoms; between 500 and 2000 atoms the truncated octahedron was their lowest energy structure followed by octahedron, truncated decahedron and Ih. In this case calculations were performed using Ino's theory with parameters from the Sutton-Chen potential. DFT calculations performed by $\mathrm{Li}$ et al. ${ }^{29}$ showed that for $\mathrm{Au}_{561}$, the order of stability was fcc, Dh and Ih. In contrast to these results, Barnard et al. ${ }^{30}$ reported that, based on a thermodynamic model, the Ih was the most stable structure at room temperature, while at temperatures comparable to our equilibrium region the $\mathrm{Dh}$ was the most stable structure. In both cases fcc was the lowest free energy structure only for cluster sizes $>15 \mathrm{~nm}$. In a global optimisation study (using the RGL potential) by Göedecker et al. ${ }^{31}$, it was found that a truncated octahedral $\mathrm{Au}$ cluster with 201 atoms was only $0.007 \mathrm{eV}$ higher in energy than a 192 atom Marks decahedral cluster. These very small differences in energy between $\mathrm{Dh}$ and fcc isomers are broadly consistent with our experimental observations.

Given the small energy difference obtained between the two principal isomers, $40 \mathrm{meV}$, the influence of the substrate needs to be considered. As described in Note 2 of the Supplementary Information, we have conducted an experimental investigation of the same isomers of $\mathrm{Au}_{561}$ but this time on an amorphous carbon support. The behaviour observed is similar: an annealing regime followed by an equilibrium regime; the fcc structure has the lowest energy; the $\mathrm{Dh}$ is, in this case, found to lie $20 \mathrm{meV}$ higher in energy. We conclude that the method reported has general applicability to different systems and that the change of support 
does not markedly alter the relative energies. Another question is: does the surface switch the relative stability of the two isomers compared with the free clusters? Without any experimental data from the gas phase one cannot be sure, but we have conducted a theoretical treatment of the substrate effect (Supplementary Note 3). This shows that a model of the carbon surface has a tendency to stabilise the fcc isomer more than it does the Dh isomer, which relates to the facet sizes in contact with the support. Thus it is possible that the favoured configuration of a free cluster could switch on the surface from Dh to fcc.

In summary, we have demonstrated a method to obtain experimentally a critical parameter in the PES of a model nanosystem. Specifically, we have reported the proportions of competing structural isomers as a function of temperature in a population of model size-selected $\mathrm{Au}_{561}$ clusters, soft-landed on amorphous silicon nitride. The approach employs atomicresolution imaging with an ultrastable heating stage in the aberration-corrected STEM. Two distinct kinds of behaviour have been identified. In the low-temperature region, from 20 to $125^{\circ} \mathrm{C}$, there is a decrease in the Dh:fcc ratio, attributed to the transformation of kinetically trapped metastable Dh into lower energy fcc structures. In the higher temperature region, from 125 to $500^{\circ}$ $\mathrm{C}$, the Dh:fcc ratio increases; the Dh isomer is repopulated because the system is in equilibrium. The measured equilibrium populations enable us to determine the energy difference between the two isomers. We find that the $\mathrm{Dh}$ isomer is $0.040 \pm 0.020 \mathrm{eV}$ higher in energy than the fcc for $\mathrm{Au}_{561 \pm 14}$. Ultimately, such quantitative parameters of the PES allow for a direct comparison with, and benchmark of, theoretical treatments and thus a new insight into the equilibrium structures and dynamics of nanosystems.

\section{Methods}

Cluster deposition. Au clusters consisting of $561 \pm 14$ atoms were produced with a magnetron sputtering gas aggregation cluster beam source ${ }^{32}$, incorporating a lateral time of flight mass filter $(M / \Delta M=20)^{33}$. The clusters were deposited onto the amorphous silicon nitride films of the heating chips in the soft-landing regime $(<2$ $\mathrm{eV} /$ atom $)^{34}$ to preserve their original atomic structure.

Electron microscopy. A JEOL 2100F STEM with spherical aberration probe corrector (CEOS) was employed for atomic-resolution imaging of the nanoclusters. The convergence angle was $19 \mathrm{mrad}$, and the inner and outer HAADF detector collection angles were $62 \mathrm{mrad}$ and $164 \mathrm{mrad}$ respectively. In situ heating was performed using a heating holder with MEMS-based heating chips (DENS Solutions). The chips consist of a metal heater coil embedded in silicon nitride, surrounded by imaging windows of amorphous silicon nitride. A current is applied to the metal coil to heat the chips: the temperature comes from the resistance measured in situ using the four-point probe method, with chip calibration performed by the supplier. The error on the temperature measurement is $5 \%$ (a potential systematic error) and the temperature stability $<1^{\circ} \mathrm{C}$. Experiments were conducted by setting the temperature to a chosen value and taking single-shot HAADF STEM atomic-resolution images of a population of clusters. The temperature was increased incrementally (from $20^{\circ} \mathrm{C}$ to $500^{\circ} \mathrm{C}$ ) and at each temperature $\geq 100$ clusters were imaged. The atomic structures of the individual clusters were then identified by comparison with multi-slice electron scattering simulations of the (unsupported) cuboctahedron, Ino-decahedron and icosahedron isomers at different orientations (polar and azimuthal) using the QSTEM package and the simulation atlas method ${ }^{19}$.

Data availability. All data is available from the authors upon reasonable request.

Received: 24 September 2017 Accepted: 12 March 2018

Published online: 03 April 2018

\section{References}

1. Topsakal, M., Aktürk, E. \& Ciraci, S. First principles study of two- and onedimensional honeycomb structures of boron nitride. Phys. Rev. B 79, 115442 (2009).
2. Szwacki, N. G., Sadrzadeh, A. \& Yakobson, B. I. $B_{80}$ fullerene: an ab intitio prediction of geometry, stability and electronic structure. Phys. Rev. Lett. 98, 166804 (2007)

3. Li, C., Guo, W., Kong, Y. \& Gao, H. First-principles study of the dependence of ground-state structural properties on the dimensionality and size of $\mathrm{ZnO}$ nanostructures. Phys. Rev. B 76, 035322 (2007).

4. Wu, X., Wei, Z., Sun, Y., Feng, Y. L. \& Liu, Q. M. Influence of the potential model parameters on the structures and potential energy surface of cobalt clusters. Chem. Phys. Lett. 660, 11-17 (2016).

5. Piotrowski, M. J., Piquini, P. \& Da Silva, J. L. F. Density functional theory investigation of 3d, 4d, and 5d 13-atom metal clusters. Phys. Rev. B 81, 155446 (2010).

6. Gruner, M. E., Rollmann, G., Entel, P. \& Farle, M. Multiply twinned morphologies of FePt and CoPt nanoparticles. Phys. Rev. Lett. 100, 087203 (2008).

7. Kumar, V. \& Kawazoe, Y. Evolution of atomic and electronic structure of Pt clusters: planar, layered, pyramidal, cage, cubic, and octahedral growth. Phys. Rev. B 77, 205418 (2008).

8. Baletto, F. \& Ferrando, R. Structural properties of nanoclusters: energetic, thermodynamic, and kinetic effects. Rev. Mod. Phys. 77, 371 (2005).

9. Campbell, C. T. The active site in nanoparticle gold catalysis. Science 306, 234-235 (2004).

10. Hernandez-Fernandez, P. et al. Mass-selected nanoparticles of $\mathrm{Pt}_{\mathrm{x}} \mathrm{Y}$ as model catalysts for oxygen electroreduction. Nat. Chem. 6, 732-738 (2014).

11. Ellis, P. R. et al. The cluster beam route to model catalysts and beyond. Faraday Discuss. 188, 39-56 (2016).

12. Cully, M. Drug delivery: nanoparticles improve profile of molecularly targeted cancer drug. Nat. Rev. Drug Discov. 15, 231 (2016).

13. Agasti, S. S. et al. Photoregulated release of caged anticancer drugs from gold nanoparticles. J. Am. Chem. Soc. 131, 5728-5729 (2009).

14. Saha, K., Agasti, S., Kim, C., Li, X. \& Rotello, V. M. Gold nanoparticles in chemical and biological sensing. Chem. Rev. 112, 2739-2779 (2012).

15. Wiesel, A. et al. Structures of medium sized tin cluster anions. Phys. Chem. Chem. Phys. 14, 234-245 (2012).

16. Barke, I. et al. The 3D-architecture of individual free silver nanoparticles captured by X-ray scattering. Nat. Commun. 6, 6187 (2015).

17. Koga, K. \& Sugawara, K. Population statistics of gold nanoparticle morphologies: direct determination by HREM observations. Surf. Sci. 529, 23-35 (2003).

18. Li, Z. Y. et al. Three-dimensional atomic-scale structure of size-selected gold nanoclusters. Nature 451, 46-48 (2008).

19. Wang, Z. W. \& Palmer, R. E. Determination of the ground-state atomic structures of size-selected Au nanoclusters by electron-beam-induced transformation. Phys. Rev. Lett. 108, 245502 (2012).

20. Wells, D., Rossi, G., Ferrando, R. \& Palmer, R. E. Metastability of the atomic structures of size-selected gold nanoparticles. Nanoscale 7, 6498-6503 (2015).

21. Wang, Z. W. \& Palmer, R. E. Experimental evidence for fluctuating, chiral-type $\mathrm{Au}_{55}$ clusters by direct atomic imaging. Nano Lett. 12, 5510-5514 (2012).

22. Wang, Z. W. \& Palmer, R. E. Direct atomic imaging and dynamical fluctuations of the tetrahedral $\mathrm{Au}_{20}$ cluster. Nanoscale 4, 4947-4949 (2012).

23. Ajayan, P. M. \& Marks, L. D. Experimental evidence for quasimelting in small particles. Phys. Rev. Lett. 63, 279-282 (1989).

24. Koga, K., Ikeshoji, T. \& Sugawara, K. Size-and temperature-dependent structural transitions in gold nanoparticles. Phys. Rev. Lett. 92, 115507 (2004).

25. Plant, S. R., Cao, L. \& Palmer, R. E. Atomic structure control of size-selected gold nanoclusters during formation. J. Am. Chem. Soc. 136, 7559-7562 (2014).

26. Weis, P., Bierweiler, T., Vollmer, E. \& Kappes, M. M. ${ }^{+}{ }^{+} u_{9}:$ rapid isomerization reactions at $140 \mathrm{~K}$. J. Chem. Phys. 117, 9293-9297 (2002).

27. Baletto, F., Ferrando, R., Fortunelli, A., Montalenti, F. \& Mottet, C. Crossover among structural motifs in transition and noble-metal clusters. J. Chem. Phys. 116, 3856-3863 (2002).

28. Wang, B., Liu, M., Wang, Y. \& Chen, X. Structures and energetics of silver and gold nanoparticles. J. Phys. Chem. C 115, 11374-11381 (2011).

29. $\mathrm{Li}, \mathrm{H}$. et al. Magic number gold nanoclusters with diameters from 1 to $3.5 \mathrm{~nm}$ : relative stability and catalytic activity for $\mathrm{CO}$ oxidation. Nano Lett. 15 , 682-688 (2015).

30. Barnard, A. S., Young, N. P., Kirkland, A. I., van Huis, M. A. \& Xu, H. Nanogold: a quantitative phase map. ACS Nano 3, 1431-1436 (2009).

31. Bao, K., Goedecker, S., Koga, K., Lancon, F. \& Neelov, A. Structure of large gold clusters obtained by global optimization using the minima hopping method. Phys. Rev. B 79, 041405(R) (2009).

32. Pratontep, S., Carroll, S. J., Xirouchaki, C., Streun, M. \& Palmer, R. E. Size-selected cluster beam source based on radio frequency magnetron plasma sputtering and gas condensation. Rev. Sci. Instrum. 76, 045103 (2005). 
33. Von Issendorff, B. \& Palmer, R. E. A new high transmission infinite range mass selector for cluster and nanoparticle beams. Rev. Sci. Instrum. 70, 4497 (1999).

34. Di Vece, M., Palomba, S. \& Palmer, R. E. Pinning of size-selected gold and nickel nanoclusters on graphite. Phys. Rev. B 72, 073407 (2005).

\section{Acknowledgements}

We thank EPSRC for financial support of the experiments. D.M.F. is grateful for financial support from the EU project NanoMILE.

\section{Author contributions}

D.M.F. conducted the experiments and analysed the data with R.F., R.E.P. initiated the experiments and supervised the work. The authors wrote the paper together.

\section{Additional information}

Supplementary Information accompanies this paper at https://doi.org/10.1038/s41467018-03794-9.

Competing interests: The authors declare no competing interests.
Reprints and permission information is available online at http://npg.nature.com/ reprintsandpermissions/

Publisher's note: Springer Nature remains neutral with regard to jurisdictional claims in published maps and institutional affiliations.

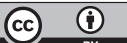

Open Access This article is licensed under a Creative Commons Attribution 4.0 International License, which permits use, sharing, adaptation, distribution and reproduction in any medium or format, as long as you give appropriate credit to the original author(s) and the source, provide a link to the Creative Commons license, and indicate if changes were made. The images or other third party material in this article are included in the article's Creative Commons license, unless indicated otherwise in a credit line to the material. If material is not included in the article's Creative Commons license and your intended use is not permitted by statutory regulation or exceeds the permitted use, you will need to obtain permission directly from the copyright holder. To view a copy of this license, visit http://creativecommons.org/ licenses/by/4.0/.

(C) The Author(s) 2018 